Relations industrielles

Industrial Relations

\title{
Managing Employment Change par Huw BEYNON, Damian GRIMSHAW, Jill RUBERY et Kevin WARD, Oxford : Oxford University Press, 2002, 342 p., ISBN 0-19-924869-9.
}

\section{Sid Ahmed Soussi}

Volume 60, numéro 2, printemps 2005

URI : https://id.erudit.org/iderudit/011729ar

DOI : https://doi.org/10.7202/011729ar

Aller au sommaire du numéro

Éditeur(s)

Département des relations industrielles de l'Université Laval

ISSN

0034-379X (imprimé)

1703-8138 (numérique)

Découvrir la revue

Citer ce compte rendu

Soussi, S. A. (2005). Compte rendu de [Managing Employment Change par HUW BEYNON, Damian GRIMSHAW, Jill RUBERY et Kevin WARD, Oxford : Oxford University Press, 2002, 342 p., ISBN 0-19-924869-9.] Relations industrielles / Industrial Relations, 60(2), 378-381. https://doi.org/10.7202/011729ar d'utilisation que vous pouvez consulter en ligne. 
support to ensure that such a link can be clearly established. This question of unions responding without a strong and committed level of state support, even under the post-1997 New Labour government, is a leitmotiv of the book but it never quite surfaces as an explicit issue in itself. Soft regulatory support appears to be developing, but it has to be underpinned by trade union intervention and resources. Hall and Terry's chapter does outline this new regulatory dimension with its piecemeal approach. However, even they acknowledge that much appears to depend on employers and their attitudes.

The question of trade union responses and employers comes to a head with two key chapters which synthesis the debate on partnership and co-operative relations with employers. Danford et al. see the development of trade union engagement with partnership as a flawed strategy due to its role in legitimating a more exploitative environment in terms of high performance work systems. They are concerned that this aspect of trade union responses may contradict the broader activities of labour and suck unions into the managerial concerns of the firm. Oxenbridge and Brown differ, arguing that there can be benefits to a closer working relationship if trade unions have a clear relation with their members and independent vision of their objectives.

If any reader wanted to understand the state of the labour movement in the United Kingdom in relation to employment change and regulation, then this book is a vital acquisition. The book brings together what can be considered to be the best research in the area. It is structured and introduced in such a way that the multi-dimensional challenge to labour is effectively described, as are the responses to such challenges. In this respect, the last chapters by Druker and Stanworth on agency labour and the Abbott on the Citizens Advice Bureau (a national and free advice body for workers and consumers) raise a new set of developments regarding 'other actors' and their role within the representation of rights. This notion of 'other actors' is becoming a major area of academic development as the question of voice is seen in broader terms: future editions of the British Journal of Industrial Relations will become much more engaging with such developments, according to its editor (Ed Heery who is one of the editors of this book as well).

If there was one missing link in the book, it would be the question of the political nature of worker representation, which has become a slightly more problematic issue given the current nature of social democracy in Britain. Yet, this is a minor point in what is otherwise a benchmark intervention on the subject of worker representation and its complexities.

Miguel MarTínez LuCio
University of Bradford School
of Management, UK

\section{Managing Employment Change}

par Huw BEYNON, Damian GRIMSHAw, Jill RuBERY et Kevin WARD, Oxford : Oxford University Press, 2002, 342 p., ISBN 0-19-924869-9.

Cet ouvrage est le produit d'un travail collectif résultant d'une collaboration entre chercheurs universitaires et institutionnels européens. Il s'agit d'une enquête basée sur sept études de cas couvrant, entre 1996 et 1999, vingttrois espaces de travail, des secteurs public et privé, situés dans le nord du Royaume-Uni. Ce type de recherche reflète une tendance lourde en Europe 
en matière de design de recherche à la fois multidisciplinaire (économistes, géographes et sociologues) et interinstitutionnelle (centres de recherche nationaux et continentaux). L'ouvrage est divisé en trois sections thématiques, mais cette répartition est plutôt formelle car c'est à travers les neuf chapitres que sont véritablement répartis les résultats de la recherche. L'objectif est d'emblée précisé : il ne s'agit pas d'expliquer les principales transformations récentes du travail mais de déconstruire les mécanismes qui ont conduit à la restructuration du marché de l'emploi dans le contexte d'une économie dont l'essence même a changé de nature sous deux impacts. Celui de la transition d'un fort partenariat en matière de relations collective du travail vers un rapport salarial individualisé fragilisant l'employé face à l'employeur; ensuite celui de la fragmentation du travail industriel résultant des transformations des structures de production (délocalisation, externalisation).

Les pertinences sociales et scientifiques invoquées sous-tendent l'ambition des auteurs pour qui il s'impose de dépasser ces poncifs trop globalisants en les repensant autrement et en s'appuyant sur des études empiriques fouillées permettant, seules, de documenter ces transformations et, surtout, les impacts de ces dernières sur les nouveaux modes d'organisation du travail humain et leurs incidences sur les cheminements de carrière et la conciliation travail-famille.

La démarche inductive des auteurs repose sur quelques constats de base. Les impacts de ces changements montrent que les mécanismes de flexibilité du travail, loin de produire les « arrangements positifs » qui les justifient en termes de conciliation travail-famille, génèrent au contraire des effets pervers sur la structure de l'emploi à l'échelle régionale. La transversalité de ces effets est renforcée par la tendance marquée du management public à «importer » les pratiques de gestion du secteur privé, dans le cadre précisément de la politique dite de nouveau management public (NMP). Un autre constat inattendu montre que les entreprises de «l'économie nouvelle n'ont fait que consolider cette tendance. Les mêmes causes produisant les mêmes effets, l'on remarque que depuis les années 1997, le recours au $N M P$ s'est accompagné de mécanismes de «flexibilité » en tous points comparables à ceux du secteur privé.

Le premier chapitre présente les entreprises choisies pour couvrir tous les secteurs d'activité affectés par les transformations du travail et de l'emploi. Ces organisations sont à peine dissimulées sous les désignations de « Bankco », « Councilco », « Healthco », «Mediaco », « Pharmco », « Retailco» et « Telecomco ». Pour chacune d'elles, l'enquête a documenté les nouveaux rôles des fonctions organisationnelles déterminantes au regard de la problématique de recherche : GOP, finances, $\mathrm{RH}$ et relations du travail, et relations avec clients et fournisseurs.

Une première série de résultats montre que, concernant les transformations du travail, même si Pharmaco se distingue des autres, les facteurs décisifs ne relèvent pas seulement du déterminisme technologique ou des pratiques de gestion, mais d'une logique plus complexe liée aux changements affectant l'environnement sociétal de la société britannique dans son ensemble. Quant aux dimensions internes (à l'entreprise) de ces transformations, les auteurs soulignent clairement l'opportunisme stratégique des décideurs (managériaux et exécutifs) mais en insistant sur sa conjugaison avec d'autres facteurs contingents comme les résistances individuelles et collectives liées aux contraintes de l'encadrement institutionnel des relations du travail.

Le reste des résultats porte sur les perceptions empiriques des employés et des gestionnaires interrogés dans 
chaque étude de cas. Il y apparaît qu'aux marchés internes de l'emploi se substituent graduellement des systèmes de gestion fondés sur une plus grande «flexibilité » de la main-d'œuvre, notamment en raison de la multiplication des interventions des agences d'intérim. Certains secteurs (Telecomco, Mediaco) sont plus marqués que d'autres (Retailco), mais la tendance est nette. De façon systématique, il en ressort un net resserrement des équipes de gestionnaires. Le tout se faisant dans un contexte de rentabilité à court terme, d'une intensité croissante des tâches et d'une réduction des coûts de la main-d'œuvre, ce dernier élément étant le plus souvent conjugué à la multiplication des contrats à durée déterminée au détriment de ceux à long terme. L'enquête souligne également l'accroissement rapide et substantiel du phénomène du contrôle et de la surveillance au travail. Son caractère transversal à tous les secteurs étudiés fait montre d'une intensification récurrente à toute la chaîne de valeur dont chaque secteur est porteur d'une forme spécifique mais fonctionnant sur la base des mêmes principes de réduction des coûts par un contrôle plus serré des tâches et des individus.

Pour les auteurs, la structure de la main-d'œuvre et de l'emploi a subi de profondes transformations. Les relations collectives du travail cèdent le pas à un rapport salarial de plus en plus individualisé sous la pression de nouveaux modes d'organisation du travail dont le caractère trans-sectoriel est avéré. Par-delà les spécificités technologiques et organisationnelles de chaque secteur, les processus mis en place sont fondés sur les mêmes principes cardinaux de flexibilité, de réduction des coûts, du contrôle et de la surveillance ainsi que de l'incontournable rentabilité à court terme. De surcroît, cette évolution fait en sorte que l'encadrement institutionnel du travail échappe de plus en plus aux politiques nationales ad hoc, le cas du Royaume-Uni étant à ce titre illustratif d'une tendance récurrente à l'échelle continentale.

Le cadre théorique des auteurs commence par se situer par rapport à l'analyse historique de Polanyi (1944) et du courant qui s'est développé dans son sillage (Harvey, 1999; Block, 1986; Granovetter, 1974, 1985) autour de "l'embeddedness of market and production relations within local social, political and cultural forms » (p. 24). Cela pour ensuite s'en démarquer en s'inscrivant dans une perspective résolument institutionnaliste, dans la droite ligne des travaux fondateurs de D. North. Ils affirment que même si l'interface complexe entre l'organisation économique et son environnement externe est d'une « importance considérable », un surdéterminisme abusif lui a été attribué dans l'analyse des transformations du travail et de la structure de la main-d'œuvre. L'illustration type en est pour eux la prétendue niponnisation des espaces de travail associée aux transferts des pratiques managériales éponymes en Angleterre et aux États-Unis notamment. C'est dans ce contexte qu'a été négligée la portée des substrats sociaux, culturels et identitaires véhiculés par ces pratiques.

S'appuyant sur l'école française de la régulation (Sorge et Maurice, 2000; Maurice et al., 1986) et les travaux de l'école néo-culturaliste, ils soulignent également la contingence déterminante des facteurs externes aux organisations, notamment ceux liés aux institutions économiques, sociales et politiques. Les mécanismes par lesquels les entreprises intègrent cette contingence s'avèrent déterminants quant à la structure du marché interne de la main-d'œuvre et de l'emploi. Culture organisationnelle et relations de pouvoir dans l'entreprise ne peuvent donc avoir de sens hors de cette contingence, malgré la prétention des organisations à implanter leurs « valeurs managériales » indépendamment des espaces sociétaux où elles s'installent et des modes de régulation économique 
de ces espaces. C'est dire toute la portée et la pertinence de l'approche choisie dans cet ouvrage dans le contexte du débat scientifique actuel où leur analyse semble résolument à contre-courant des tendances dominantes, notamment en sciences administratives.

Sid AHMed Soussi

Université du Québec en Outaouais

\section{From Consent to Coercion: The Assault on Trade Union Freedoms, $3^{\text {rd }}$ edition \\ by Leo PANITCH and Donald Swartz, Aurora, Ont.: Garamond Press, 2003, 270 pp., ISBN 1-55193-049-8.}

American trade union advocates tend to see Canada as the Promised Land they would like to reach at home. Canadian workers have 30 percent union density, a level not seen in the United States since the 1950s. Unions can gain recognition based on signed membership cards, or at worst, through elections held in five days, not through NLRB election campaigns that give companies several weeks of "employer free speech" rights to smash workers' organizing efforts. Canada's "Rand Formula" grants dues payments to unions from all represented workers, in contrast to union-weakening "right-to-work" laws in the United States requiring unions to represent non-paying free riders.

The threat of permanent replacement looms over any strike decision by American workers, while permanent replacement of striking workers is unlawful in Canada. In their North American version of European social democracy, Canadians enjoy national health insurance while U.S. workers deal with a patchwork of employer-based health plans that provokes constant strife, strikes, and cuts in benefits.

Leo Panitch and Donald Swartz throw a bucket of cold water on this wide-eyed American view of labour affairs north of the border. Their third edition of From Consent to Coercion: The Assault on Trade Union Freedoms carefully details many ways in which Canadian labour law and practice run afoul of international standards on labour rights, and how Canada's
Supreme Court has interpreted the nation's Charter of Rights and Freedoms to constrict workers' freedom of association.

In the time of NAFTA and a looming Free Trade Agreement of the Americas, crafting a social dimension in trade pacts requires critical understanding of labour law and practice among trading partners. This book is an important contribution to labour policy debates for the spotlight it turns on Canada, driving home the insight that no country comes to the labour rights debate with totally clean hands.

The most common breach of workers' rights in Canada takes the form of back-to-work legislation putting an end to strikes with government-imposed settlements instead of terms negotiated between bargaining parties. What started as exceptional action in the post World War II period (the age of "consent" in the title) has become standard operating procedure for federal and provincial governments. In the quarter-century after 1950, governments ended strikes with back-to-work legislation 32 times. The number rose to 115 imposed strike settlements from 1975 to 2002, reflecting the turn to "coercion."

Panitch and Swartz analyze the trend not just by looking at the numbers. They tell an important story about political and economic dynamics that drove the move from consent to coercion. The story has ironies, too: Chapter 3 starts with a ringing quote from young Pierre 